\title{
Malingering in Forensic Psychiatry Assessment
}

Seyed Mehdi Saberi ${ }^{1}$, Mohammad-Reza Karimi ${ }^{1}$, Ardeshir Sheikhazadi ${ }^{2}$, Mazaher Ghorbani ${ }^{2}$, Ali Pasha Meysamie ${ }^{3}$, Zeynab Nasri Nasrabadi ${ }^{2}$ and Sayed Mahdi Marashi*

${ }^{1}$ Department of Psychiatry of Legal Medicine Organization, Tehran, Iran

${ }^{2}$ epartment of Forensic Medicine and Toxicology, Tehran University of Medical Sciences, Tehran, Iran

${ }^{3}$ Department of Community Medicine, Tehran University of Medical Sciences, Tehran, Iran

\begin{abstract}
Malingering and deceitfulness are of the greatest issues facing a forensic examiner. Salient point in the malingerers is that creating deliberate and conversant physical and mental symptoms usually is based on an external motivation. Most cases consciously express subjective and ambiguous symptoms; and when represent symptoms of a definite psychiatric condition, usually claim symptoms sometimes disappeared and evade to assent remedial plans. It is necessary to be always aware of the possibility of faking symptoms in forensic cases to make appropriating decision and avoid blunder in court.
\end{abstract}

Keywords: Crime; Malingering; Deception; Claim; Forensic psychiatry; Evaluation

\section{Introduction}

One of the most considerable issues facing a forensic examiner is the concept of detecting deceitfulness and malingering, particularly in the forensic psychiatry setting [1]. Salient point in malingerers is that an external impetus for creating conversant physical and mental symptoms almost in all cases exist [2]. Most malingerers consciously express subjective and obscure symptoms; when represent symptoms of a definite psychiatric condition, usually claim symptoms sometimes disappeared and evade to assent remedial plans [1]. In the most studies diagnostic tests have been evaluated, however studies on faked psychiatric symptoms slightly implemented up to now [2].

Malingering symptoms as mental disorders, cognitive impairments and clinical syndromes were compared in forensic and non-forensic cases [3]. Female malingerers in this study had simulated medical symptoms about twofold more than male participants (50\% vs. $25 \%$ ) but cognitive deficits had a greater percentage in males $[22.7 \%$ vs. 6.4\%] than their female coequals. Another finding in this study was that in non-forensic setting participants may fake mental disorders more, but in a forensic setting the most cases may feign medical symptoms and cognitive impairment [3].

Cornell and et al. in their study on clinical defendants referred for evaluation of criminal responsibility showed that in a pretrial, outpatient evaluation, the incidence of malingering was about $8 \%$. In comparison of malingerers and genuine psychotics, malingerers were less educated and were more charged with criminal act against persons rather than financial crimes. Sexual offenses, robbery and assault were the most common causes of juridical order to evaluate standing trial. Most of malingerers also had a history of several prior arrested because of serious crimes against people. Auditory hallucination and exaggerated behavior were the most common imitating symptoms whereas disproportionate affect, neologism, non-abstract thinking and impaired personal hygiene seldom been faked. Some were representing clear symptoms have dealings with mental disorder, such as suicidal image and visual hallucinations [4].

The relevance among criminal charges, diagnosis and ability to stand trial and criminal liability was evaluated by Cochrane and et al. In evaluation of 1710 defendants they showed that about $24 \%$ of all cases had an antisocial personality disorder, $32 \%$ having one psychotic disorders and the incidence of malingering was $11 \%$. Malingerers were simulating symptoms of psychosis, cognitive impairment, and multiple personality disorder and memory impairment. Those charged with kidnapping and robbery had higher rates of malingering $(23 \%$ and $17 \%$ respectively) and surprisingly, those charged with murder had the least rates of malingering (4\%). They determined that kidnapping, state crimes and drug related crimes need more cognitive capability to make proper mapping. Therefore these complex crimes rarely correlate with mental illness, incompetence and insanity. Low rate of malingering among those charged with murder concluded to be probably the consequence of high stakes involved and more pressure on defendants to feign symptoms; however the influence of racial differences proposed to be evaluated on malingerers [5].

Kucharski and et al. used The Minnesota Multiphasic Personality Inventory (MMPI-2) to evaluate malingering in two groups of defendants with, and without preexisting history of psychiatric hospitalization as probable honest and suspected malingerers, referred for assessment of competence to stand trial or criminal responsibility. They showed that unusual hallucinatory experiences, unusual symptom presentation and global assessment of functioning represent the best discriminators and certain diagnosis can be made in $90 \%$ of malingerers. Often, malingerers' level of functioning in the past year, based on the interview with their families, does not match with their mental symptoms [6].

In another study by Poythress and et al. the Personality Assessment Inventory (PAI) was used to assess symptoms of major mental illness in a group of prisoners (as general population) that was ordered to answer the questions loyally as the 'honest' group, and another group of prisoners that was ordered to simulate mental disorder

${ }^{*}$ Corresponding author: Sayed Mahdi Marashi, Department of forensic medicine and toxicology, Tehran University Of Medical Sciences, 16 azar avenue, Tehran, Iran.Tel: +982166405588; E-mail: marashi_m@razi.tums.ac.ir

Received September 21, 2011; Accepted December 02, 2011; Published December 05, 2011

Citation: Saberi SM, Karimi MR, Sheikhazadi A, Ghorbani M, Meysamie AP, et al (2011) Malingering in Forensic Psychiatry Assessment. J Forensic Res S2:006. doi:10.4172/2157-7145.S2-006

Copyright: (c) 2011 Saberi SM, et al. This is an open-access article distributed under the terms of the Creative Commons Attribution License, which permits unrestricted use, distribution, and reproduction in any medium, provided the original author and source are credited. 
symptoms as 'known malingerer' group and compared them with a mental health unit participants clinically judged as 'malingerers' and 'non-malingerers'. Psychopathic characteristics were evaluated via psychopathic personality inventory (PPI). They revealed that correlations between the PPI and the feigning indices of depression, mania, paranoia and schizophrenia were not statistically significant. Therefore concluded that despite the expectance, participants with innate psychopathic personality disorder are not more successful at simulate symptoms of a major mental illness than the others. [7].

Mittenburg and the colleagues observed the prevalence of malingering and symptom exaggeration using neuropsychological examinations. In these way 131 members of the American Board of Clinical Neuropsychology, from different geographically regions, shared their practice on demographics, source of the order to examination, base rates of probable malingerers and the methods used to make the diagnosis. They reported 33531 examinations in one year survey, of whom $29 \%$ of 6371 cases of personal injury, 30\% of 3688 cases of disability, $19 \%$ of 1341 criminal cases and $8 \%$ of 22131 medical cases involved symptom exaggeration or malingering. The prevalence estimates among different geographic regions were not differing significantly. In personal injury and disability cases, referral by the insurer was related to higher rates of probable malingering; higher prevalence of malingering was observed in criminal cases that referred by the prosecution as well. Specialists involved in this study classically used multiple methods including standard inventories and general psychiatric interviews for assessing the probability of symptom exaggeration [8].
We proposed that it is necessary for every psychologist or psychiatrist to be aware of the possibility of faking symptoms especially in forensic cases, to make appropriating decision and avoid blunder in court.

\section{References}

1. Hall HV, Poirier JG (2000) Detecting malingering and deception: Forensic distortion analysis. CRC Press. page 3-5.

2. Sellbom M, Ben-Porath YS,Graham JR, Arbisi PA, Bagby RM (2005) Susceptibility of the MMPI-2 clinical, restructured clinical (RC), and content scales to overreporting and underreporting. Assessment 12: 79-85.

3. Rogers R, Salekin RT, Sewell KW, Goldstein A, Leonard K (1998) A comparison of forensic and non-forensic malingerers: A prototypical analysis of explanatory models. Law and human behavior 22: 353-367.

4. Cornel DG, Hawk GL (1989) Clinical presentation of malingeres diagnosed by experienced forensic psychologist. Law and human behavior 13: 375-383.

5. Cochrane RE, Grisso T, Frederick RI (2001) The relationship between criminal charges, diagnoses and psycholegal opinions among federal preterial defendants. Behavioral sciences and the law 19: 565-582.

6. Kucharski LT, Ryan W, Vogt J, Goodloe E (1998) Clinical symptom presentation in suspected malingerers: An empirical investigation. J Am Acad Psychiatry Law 26: 579-585.

7. Poythress NG, Edens JF, Watkins MM (2001) The Relationship Between Psychopathic Personality Features and Malingering Symptoms of Major Mental Illness. Law and Hum Behav 25: 567-582.

8. Mittenberg W, Patton C, Canyock EM, Condit DC (2002) Base rate of malingering and symptom exaggeration. J Clin Exp Neuropsychol24: 1094 1102.

This article was originally published in a special issue, Forensic Medicine handled by Editor(s). Dr. Kate Barnes, University of Derby, UK 